\title{
Interplay of Nutrients and Muscle Myokines on the Preventive Health for Obesity Disorders
}

ISSN: 2578-0263

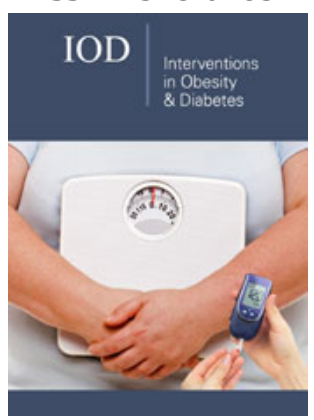

*Corresponding author: Rajasekhar Kali Venkata, Director of Physical Education and sports sciences, India

Submission: 1-in August 20, 2019

Published: 制September 04, 2019

Volume 3 - Issue 2

How to cite this article: Rajasekhar $\mathrm{K} \mathrm{V}$, Praveen. Interplay of Nutrients and Muscle Myokines on the Preventive Health for Obesity Disorders. 3(2). IOD.000558.2019. DOI: 10.31031/IOD.2019.03.000558

Copyright@ Rajasekhar Kali Venkata. This article is distributed under the terms of the Creative Commons Attribution 4.0 International License, which permits unrestricted use and redistribution provided that the original author and source are credited.

\author{
Rajasekhar Kali Venkata* and Praveen \\ ${ }^{1}$ Director of Physical Education and Sports Sciences, University of Hyderabad, India \\ ${ }^{2}$ Associate Professor, Department of Physical Education, Pondicherry University, India.
}

\section{Opinion}

Physical exercise seems to be an effective tool in enhancing the functional health of the various systems of the body and the consequent health status of individuals. Physical inactivity could foster for flaring up of inflammatory mechanisms that might affect different tissues negatively and could lead to severe debilitating health conditions. Though the physical exercise could play pivotal role in securing the preventive health, the science of disease reiterates that there seems a systematic inter connectivity among molecular responses that make the bioenvironment so dynamic, may respond differently and or in inter-connected manner to several epigenetic factors like exercise, nutrition, stress, environment etc. and could show rhetoric impact on the genetic expression, thereby influencing the preventive health of individuals. This rhetoric explains the need of studying the interactive epigenetic effects and consequent responses. Muscle secretum consisting of myocytes, myotubules and other skeletal muscle tissue environment produces cytokine like substances called myokines, that may initiate the cross talk among the various tissues of the body acting in autocrine, paracrine and endocrine manner. Several hundreds of such myokines are identified by researchers and attributed with different functions through the control of the signaling cascades of the metabolism. Some myokines may act directly as signaling molecules while some other are seen as co-factors for other signaling molecules thereby effecting changes in the bioenvironment of the individuals. These exercise factors may be released by the skeletal muscles differently depending on the intensity, duration and type of exercise undertaken. Aerobic and anaerobic exercises undertaken at different intensities cause for the secretion of different myokines which might bring different signaling effects and cause for different changes. Hence, aerobic running might cause for the secretion of certain types of myokines whereas the high intensity anaerobic running could cause for certain other myokine secretion. Resistance training showed different myokines released from the skeletal muscle. The physiology of muscle fibers may also influence the type of myokine secretion and its quality. Different muscle fibers may cause to produce different myokines. Exercise has an innate effect that would foster bioenvironment so favorably, to equip higher readiness for the next bout of exercise program by bringing several super compensation effects. These super compensation effects of exercise may be brought by the secretion of myokines which would influence the metabolic cascades for the above purpose. Higher exercise potential of the individuals may be achieved through regular bouts of physical training that is conducted very scientifically that would cause for several physiological changes. The readiness for higher exercise potential indicates the higher functional health of the different organs of the body and this would mostly be accompanied by higher order preventive health status for the individuals. This readiness may be achieved by enhanced anti-inflammatory status, enhanced anti-oxidative status, better arterial endothelial restitution, enhanced angiogenesis, increased substrate super compensation, enhanced positive protein turnover, enhanced glucose tolerance and insulin sensitivity, enhanced lipolytic cascades, enhanced mitochondrial enzymatic reactions, enhanced brain and hormonal axes and several such positive effects. Myokines or exercise factors might influence to bring these conditions, generally not in isolation but in combination and also release of different myokines are observed as a result of exercise program. 
The pathophysiology of obesity and the consequent disorders indicate that the adipose tissue causes for higher inflammation which is linked to inactivity. Several adipokines are produced by the adipocytes, that are also responsible for several signaling cascades that would cause both inflammatory and anti-inflammatory effects. Under the hyper obese conditions, the pro-inflammatory mechanisms far exceed the anti-inflammatory mechanisms causing several obesity related disorders. Inactivity also enhances this pro inflammatory environment in adipose tissue especially in the white adipose tissue. Meta inflammation prevails due to the excess presence of fat and consequently insulin resistance develops causing increase in inflammatory conditions that would bring excessive presence of macrophages, $\mathrm{T}$ lymphocytes, mast cells etc. Chronic insulin resistance could lead to Diabetes mellitus. There are several other possible mechanisms in which the obesity or excessive fatness would make individuals to be prone for several other diseases like kidney failure, hypercholesterolemia, atherosclerosis etc. Added inactivity to obesity could accelerate these inflammatory processes leading to several health complications including immune loss. There are several myokines that establish cross talk to the adipose tissue and bring changes in the status of adipose tissue metabolism. Myokine PGC- $1 \alpha$ (Peroxisome proliferator-activated receptor gamma co-activator 1-alpha may enhance the mitochondrial biogenesis and thereby augment the aerobic metabolism of fats and also through the activation of the AMPK signaling this exercise factor also may cause for the augmented insulin sensitivity and responsiveness. Secretion response of myokine IL-15 (Interleukin-15) may be muscle fiber type specific, the myokine has been linked to augmenting the lipid oxidation through the enhancement of expression of the gene related to PPARD (peroxisome proliferator activated receptor delta) and this induces for the enhanced muscle metabolism. Another myokine which is linked to the glucose metabolism and insulin sensitivity is FGF21 (Fibroblast Growth Factor 21). Another potent myokine is Irisin, which has been identified with several metabolic responses. Irisin is found as an important exercise factor that has the ability to convert the WAT (White Adipose Tissue) to BAT (Brown Adipose Tissue) and this augments the energy metabolism naturally. ANGPTL (Angiopoietin-like protein) which is secreted by skeletal muscles during the high intensity contractions has the ability to control the serum triglycerides by enhancing the lipolytic enzyme activity in adipose tissue. There are several such myokines which may be linked to the glucose and fat metabolism. Myokines secreted from muscle during the muscular contraction or exercise are mostly of protein or proteoglycan peptide substances and are released from the muscle into the circulation. Though the mechanism by which the secretion of muscle myokines happen during the muscle contraction is still a debated concept, but the general understanding is that the skeletal muscle myotubules could produce these myokines from the muscle tissue itself may be through lysis or through consolidation or by both. Hence, the muscle health in terms of nutrition may influence potently in the production of these myokines in terms of quality and also the quality. Nutritional status of the muscle environment along with the nutritional health of other tissues which mighty effect cross talk with the skeletal muscle, like adipose tissue might bring significant influence on the production of the muscle myokines during the muscular contraction. Several studies of nutrigenomics confirmed the direct influence of the nutrients like vitamins, minerals which may act on the epigenetic marks of the genes producing the methylation and other gene expression processes. These direct effects of the minerals, and other nutrients may be as co factors of the signalling cascades or else as direct factors that might directly influence the histone cites of the genes. Since, the exercise factors or the myokines have been recognised as very influencing and potent factors in initiating the cross talk among various tissues of the body, it would be very rational to understand the inter play effect of the myokines and the nutrients.

More and more scientific investigations are necessary in this regard to bring out the interplay effects of myokines and nutrients. It is identified that the nutritional status and the present diet could influence the secretion and circulation of FGF21 myokine, and this may be due to the epigenetic effects of nutrition. This myokine has influence on the glucose metabolism and insulin sensitivity. Some nutrients, especially phytochemicals could bring palpable effect on the anti-oxidative environment of the muscles and could very well influence on the secretion of myokines. Vitamin D may also influence on the muscle metabolism and also on the secretion of certain myokines, especially the myokine IL-6. It can be acknowledged that there may be scientific interplay connection between the secretion quality and quantity of myokines during the physical exercise from the skeletal muscles and the nutritional status of not only the skeletal muscles and also those tissues which involve in cross talk with the skeletal muscles like adipose tissue.

\section{References}

1. Allen DL, Hittel DS, McPherron AC (2011) Expression and function of myostatin in obesity, diabetes, and exercise adaptation. Med Sci Sports Exerc 43(10): 1828-1835.

2. Almuraikhy S, Kafienah W, Bashah M, Diboun I, Jaganjac M, et al. (2016) Interleukin-6 induces impairment in human subcutaneous adipogenesis in obesity associated insulin resistance. Diabetologia 59(11): 24062416.

3. Girgis CM, Clifton Bligh RJ, Hamrick MW, Holick MF, Gunton JE (2013) The roles of Vitamin D in skeletal muscle: Form, function and metabolism. Endocrine Reviews 34(1): 33-83.

4. Hoffmann C, Weigert C (2018) Skeletal muscle as an endocrine organ: The role of myokines in exercise adaptations. Cold spring Harbor perspectives in medicine 7(11): pii: a029793.

5. Huh JY, Dincer F, Mesfum E, Mantzoros CS (2014) Irisin stimulates muscle growth-related genes and regulates adipocyte differentiation and metabolism in humans. Int J Obes 38(12): 1538-1544.

6. Ikeda SI, Tamura Y, Kakehi S, Sanada H, Kawamori R, et al. (2016) Exercise-induced increase in IL-6 level enhances GLUT4 expression and insulin sensitivity in mouse skeletal muscle. Biochem Biophys Res Commun 473(4): 947-952.

7. Lee JH, Jun HS (2019) Role of myokines in regulating skeletal muscle mass and function. Frontiers in Physiology 10:42.

8. Joo Young Huh (2018) The role of exercise-induced myokines in regulating metabolism. Arch Pharm Res 41(1): 14-29. 
9. Knudsen JG, Murholm M, Carey AL, Bienso RS, Basse AL, et al. (2014) Role of IL-6 in exercise training- and cold induced UCP1 expression in subcutaneous white adipose tissue. PLoS ONE 9(1): e84910.

10. Leal LG, Lopes MA, Batista ML (2018) Physical exercise induced myokines and muscle-adipose tissue cross talk: A review of current knowledge and the implications for health and metabolic diseases. Front Physiol.

11. Rai M, Demontis F (2016) Systemic nutrient and stress signalling via myokines and myometabolites. Annu Rev Physiol 78:85-107.

12. Moreno Navarrete JM, Ortega F, Serrano M, Guerra E, Pardo G, et al.
(2013) Irisin is expressed and produced by human muscle and adipose tissue in association with obesity and insulin resistance. J Clin Endocrinol Metab 98(4): E769-E778.

13. Roy P, Chowdhury S, Roy HK (2018) Exercise induced myokines as emerging therapeutic agents in colorectal cancer prevention and treatment. Future Oncol 14(4): 309-312.

14. Vadala G (2018) Cross talk between muscle and cartilate: The myokine irisin attenuates osteoarthritis related cartilage degeneration. Orthopaedic Proceedings 100-B, supp_15. 